\title{
Exploring the potential and limitations of genotyping-by- sequencing for SNP discovery and genotyping in tetraploid potato
}

\begin{tabular}{|c|c|}
\hline Journal: & Genome \\
\hline Manuscript ID & gen-2017-0236.R1 \\
\hline Manuscript Type: & Article \\
\hline Date Submitted by the Author: & 26-Jan-2018 \\
\hline Complete List of Authors: & $\begin{array}{l}\text { Bastien, Maxime; Université Laval, Département de phytologie } \\
\text { Boudhrioua, Chiheb; Université Laval, Département de phytologie } \\
\text { Fortin, Gabrielle; Université Laval, Département de phytologie } \\
\text { Belzile, François; Université Laval, Département de phytologie }\end{array}$ \\
\hline $\begin{array}{r}\text { Is the invited manuscript for } \\
\text { consideration in a Special } \\
\text { Issue? : }\end{array}$ & N/A \\
\hline Keyword: & $\begin{array}{l}\text { genotyping-by-sequencing, potato, complexity reduction, genome-wide } \\
\text { association mapping, marker-assisted genomics }\end{array}$ \\
\hline
\end{tabular}

\section{SCHOLARONE ${ }^{m}$ \\ Manuscripts}


1 Maxime Bastien ${ }^{1}$, Chiheb Boudhrioua ${ }^{1}$, Gabrielle Fortin ${ }^{1}$, and François Belzile ${ }^{1 *}$

2

4 Exploring the potential and limitations of genotyping-by-sequencing for SNP

5 discovery and genotyping in tetraploid potato

6

7

8

$9{ }^{1}$ Département de Phytologie and Institut de Biologie Intégrative et des Systèmes (IBIS),

10 Université Laval, Quebec City, Quebec, Canada G1V OA6

11

12

$13{ }^{*}$ Corresponding author

14 Email: francois.belzile@fsaa.ulaval.ca

15 Phone: 418-656-2131 \#5763

16

17 Accepted for publication in Genome 


\section{Abstract}

20 Genotyping-by-sequencing (GBS) potentially offers a cost-effective alternative for SNP

21 discovery and genotyping. Here, we report the exploration of GBS in tetraploid potato.

22 Both ApeKI and Pstl/Mspl enzymes were used for library preparation on eight diverse 23 potato genotypes. ApeKI yielded more markers than Pstl/Mspl but provided a lower read 24 coverage per marker, resulting in more missing data and limiting effective genotyping to 25 the tetraploid mode. We then assessed the accuracy of these SNPs by comparison with 26 SolCAP data (5,824 data points in diploid mode and 3,243 data points in tetraploid 27 mode) and found the match rates between genotype calls was $90.4 \%$ and $81.3 \%$, 28 respectively. Imputation of missing data did not prove very accurate due to incomplete 29 haplotype discovery, suggesting caution in setting the allowance for missing data. To 30 further assess the quality of GBS-derived data, a genome-wide association analysis was 31 performed for flower color on 318 clones (with ApeKI). A strong association signal on 32 chromosome 2 was obtained with the most significant SNP located in the middle of the 33 dihydroflavonol 4-reductase (DFR) gene. We conclude that an appropriate choice of 34 enzyme for GBS library preparation makes it possible to obtain high-quality SNPs in 35 potato and will be helpful for marker-assisted genomics.

37 Keywords: genotyping-by-sequencing, potato, complexity reduction, genome-wide 38 association mapping, marker-assisted genomics. 
41 Introduction

42

43 The use of molecular markers derived from next-generation sequencing (NGS) has

44 begun to revolutionise the way plant genetic research is being conducted. Such

45 developments have been slower in potato than other species due to the complex nature

46 of its autotetraploid genome (Mehra et al. 2015; Hardigan et al. 2016). The most popular

47 NGS platform to this day is the Infinium 8303 Potato Array developed in 2012 by Felcher

48 et al. More recently, a subset of 4,179 SNPs from this array was included in the

49 development of a larger array (SolSTW) which comprises 19,747 SNPs (Vos et al.

50 2015). These arrays have been used for different purposes, such as fingerprinting (Bali

51 et al. 2016), pedigree analysis (Endelman et al. 2017), linkage mapping (Hackett et al.

52 2014; Manrique-Carpintero et al. 2015; Massa et al. 2015; Muktar et al. 2015; Obidiegwu

53 et al. 2015; Bourke et al. 2016; Endelman and Jansky 2016; Rak et al. 2017), diversity

54 analysis (Kolech et al. 2016) and linkage disequilibrium analysis (Vos et al. 2017).

55 However not all of these SNP markers can actually be used successfully in a given

56 experiment as they may not be polymorphic or may present a low minor allele frequency

57 (MAF) in a given population. For instance, a study based on the Infinium 8303 array

58 found 6,373 usable SNPs in a diploid calling mode and 3,763 usable SNPs in a

59 tetraploid calling mode within a diversity panel comprising 250 clones (Hirsch et al.

60 2013). Another work conducted on a collection of 350 tetraploid cultivated potato

61 varieties found 4,239 usable SNP markers, in tetraploid mode, after removing SNP

62 markers that mapped to multiple locations and SNP markers having more than $20 \%$

63 missing data as well as SNP markers whose minor allele frequency was less than $5 \%$ 
64 (Esnault et al. 2016). Similarly, the number of SNP markers available in various data

65 sets from a panel of 537 varieties using the SolSTW array varied from 5,282 to 14,530

66 (Vos et al. 2015, 2017).

67 Another drawback of such highly parallel SNP genotyping platforms is that they typically

68 carry a high cost per sample for first-time or small-scale users. In contrast, approaches

69 that enable the simultaneous discovery and genotyping over the entire genome are

70 becoming an appealing alternative. For instance, an optimised RAD-seq approach was

71 reported in potato (Jiang et al. 2016). Similarly, genotyping-by-sequencing (GBS)

72 approaches offer a highly cost-effective alternative for simultaneous SNP discovery and

73 genotyping (Elshire et al. 2011; Poland et al. 2012; Sonah et al. 2013). Moreover, GBS

74 provides other advantages such as reduced sample handling, few PCR \& purification

75 steps, no DNA size fractionation, efficient barcoding system and ease of scalability. The

76 first work labelled as GBS in potato was conducted by Uitdewilligen et al. (2013). The

77 targeted-GBS method used in this work was atypical in that complexity reduction was

78 achieved through in-solution hybridisation targeting only 807 genes. This resulted in a

79 very large number of biallelic SNPs $(105,812)$ enjoying deep read coverage and 80 accurate genotype assignment but all these SNPs resided within only 807 genic regions.

81 Recently, Sverrisdóttir et al. (2017) reported a more typical GBS approach where 82 complexity reduction was achieved strictly through enzymatic digestion (ApeKI).

83 However, rather than calling actual genotypes at each SNP locus, they simply estimated 84 variant allele frequencies at each SNP. No assessment of the accuracy of genotype 85 calls made from GBS data was provided.

86 In the present investigation, we explore the use of GBS with standard complexity 87 reduction achieved through enzymatic digestion (ApeKI or $\mathrm{Pstl} / \mathrm{Mspl}$ ) in potato and 
88 devise a python script to call genotypes in diploid or tetraploid mode. We assess the 89 accuracy of these GBS-derived SNPs by comparison with SoICAP data and explore the 90 use of imputation for filling gaps in GBS data. Finally, we demonstrate the quality of 91 GBS-derived datasets by performing an association mapping for flower color using GBS 92 data among a collection of over 300 potato clones representing the diversity of an 93 Eastern Canadian breeding program.

94 95 


\section{Material and Methods}

\section{$98 \quad$ Plant Material}

99 A set of eight diverse potato genotypes (Set A) of different origins was used for 100 restriction enzyme tests and optimization of SNP-calling parameters. The set included

101 three cultivars from eastern Canada (Conestoga, Envol and Roselys), two cultivars from 102 the United States (Eva and Russet Burbank) as well as three cultivars from Europe 103 (Argos, Divina and Hindenburg). A collection of 375 clones (Set B) representing the 104 extent of diversity present in a public potato breeding program in the province of Quebec 105 (Canada) was used to conduct phylogenetic analyses and genome-wide association 106 mapping (GWAM). This collection comprised advanced breeding clones, landraces, 107 registered varieties and ten accessions of wild potato species (S. phureja, $S$.

108 cardiophyllum, S. sparsipilum and S. stenotonum). A subset of 52 genotypes from this 109 collection (Set B2) was selected for comparison of GBS data with the Infinium 8303

110 Potato Array. Among these, 49 are registered varieties and three are advanced breeding 111 lines.

\section{DNA Extraction, Library Preparation and Sequencing}

114 DNA was extracted from $50 \mathrm{mg}$ of fresh young leaves using the DNeasy 96 Plant kit 115 (Qiagen, cat. no. 69181) following the manufacturer's protocol. DNA was quantified 116 using a Thermo Scientific Nanodrop 8000 spectrophotometer (Wilmington, DE). DNA 117 concentrations were normalized to $10 \mathrm{ng} / \mu \mathrm{l}$ and subsequently used for library 118 preparation. For optimization of the method (Set A), eight potato samples were 
119 sequenced as part of a 48-plex GBS library. The GBS libraries were prepared with

120 enzymes that have been used for GBS library preparation in several species: ApeKI as

121 per Elshire et al. (2011) and Pstl/Mspl as per Poland et al. (2012) with minor

122 modifications described in Sonah at al. (2013). For Set B (375 clones), three 96-plex and

123 one 87-plex ApeKI libraries were prepared according to the GBS protocol described by

124 Elshire et al. (2011). For all libraries, single-end sequencing was performed on an

125 Illumina HiSeq2000 (at the McGill University-Génome Québec Innovation Center in

126 Montreal, QC, Canada).

127

128 Processing of Illumina Raw Sequence Read Data and SNP Calling

129 The IGST-GBS pipeline described by Sonah et al. (2013) was used for data processing

130 of Illumina 100-bp reads and alignment on version 4.03 of the genome (Sharma et al.

131 2013). Two dosage models were used in this work. A diploid model with three marker

132 classes for each SNP (AA, AB and $B B$ ) was used for all analyses. The minimum number

133 of reads for genotype calls to be kept was 11. Four levels of tolerance for missing data

134 were compared for set $A(\leq 50, \leq 25, \leq 12.5$ or $0 \%)$, while for Sets B and B2, markers with

135 more than $20 \%$ missing were excluded from the dataset using vcftools version 0.1 .7 and

$136 \quad 0.1 .13$ (Danecek et al. 2011).

137 For the genotypic data derived from Set $\mathrm{A}$ with the use of $A p e K I$ or Pstl/Mspl, a

138 tetraploid model with five marker classes (AAAA, AAAB, AABB, ABBB and BBBB) was

139 also used. Here, genotype calls were conducted using the default parameters of

140 Freebayes v.0.9.14 (Garrison and Gabor 2012). In this case, the ploidy level was set to

141 four and a minimum of three reads supporting an alternate allele was required to call a 
142 polymorphism. An in-house Python script was then used to filter out markers based on

143 read depth. Eleven reads per site per genotype were used to call homozygotes (Simko

144 2004) while 53 reads were deemed sufficient to distinguish between the three types of

145 heterozygotes (AAAB, AABB, ABBB). Two filters were thus compared for genotype

146 calling: a relaxed filter requiring 11 reads per clone to call all genotypic classes and a

147 stringent filter requiring 11 reads to call homozygotes (AAAA \& BBBB) and 53 reads to

148 distinguish the heterozygotes ( $A A A B, A A B B \& A B B B)$. Marker datasets were then

149 submitted to further filtration using the vcffilter command of vcflib

150 (https://github.com/ekg/vcflib) to assess the impact of the proportion of missing data

151 tolerated per marker on marker number and depth. Again, four levels of missing data

152 were compared in the tetraploid model: $\leq 50, \leq 25, \leq 12.5$ or $0 \%$. Finally, markers with a

153 minor allele count inferior to 2 were excluded from the dataset.

154

155 Comparison of GBS and Infinium 8303 Potato Array SNP calls

156 Genotype calls reported by Hirsch et al. (2013) were used as a reference to assess the

157 accuracy of GBS SNP calls obtained for shared clones in this work (Set B2). Three

158 measurements of genotype accuracy were conducted: GBS-derived diploid genotype

159 calls, GBS-derived tetraploid genotype calls, and imputed diploid genotype calls. These

160 imputed genotype calls were obtained using Beagle v.4.0 (Browning and Browning

1612007 ) to impute missing data at three different levels: $\leq 20 \%, \leq 50 \%$ and $\leq 80 \%$ missing 162 data. 


\section{Genome-wide association mapping}

165 SNP data obtained in diploid mode were used to study the population structure of set B.

166 First, indels and SNPs with more than two alternate alleles, more than $20 \%$ missing data

167 or a minor allele frequency inferior to $5 \%$ were removed from the datasets. The

168 remaining markers were used to characterize population structure using a Bayesian

169 clustering approach implemented in FastStructure algorithm (Raj et al. 2014).

170 An association mapping analysis for flower pigmentation, assessed in a field trial in 2014

171 as white vs any color, was performed using the Genomic Association and Prediction

172 Integrated Tool (GAPIT version 2) (Tang et al. 2016). As GBS had failed for one of the

173 clones while phenotypic information was missing for 46 clones, these clones and the ten

174 wild accessions were removed from the dataset. Thus, a total of 318 clones were used

175 to perform association mapping. A mixed linear model $(\mathrm{Q}+\mathrm{K}$ model) taking into account

176 both population structure ( $Q$ matrix) and relative kinship (K matrix) was used. The $Q$

177 covariate and the kinship matrix calculated were used to determine relatedness among

178 individuals and eliminate false positives. The $q$-values for assessing the significance of

179 marker-trait associations were calculated based on their corresponding $p$-values (Story

180 and Tibshirani 2003). The $q$-value is a measure of significance in terms of the false

181 discovery rate similar to the $p$-value that relates to the false positive rate. This approach

182 limits the number of false positive results, while offering a more liberal criterion than the

183 Bonferroni correction factor. A $q$-value of 0.1 was used as a significant association

184 threshold (Lee et al. 2002; Benjamini et al. 2005). 


\section{Results}

\section{Comparison of ApeKI and Pstl/Mspl enzyme combinations}

189 The first series of analyses was conducted to compare GBS datasets generated using a 190 5-base (ApeKI) and a 6-base/4-base (Pstl/Mspl) restriction enzyme combination as well 191 as the diploid and tetraploid calling modes. Eight diverse potato genotypes (Set A) were 192 used to produce both ApeKI and Mspl/Pstl GBS libraries. Sequencing of the GBS 193 libraries on an Illumina HiSeq2000 yielded approximately 19.1 million reads and 19.6

194 million reads in total for the 8 potato genotypes with ApeKI and Pstl/Mspl restriction195 enzyme combinations, respectively (Table 1). Sorting of raw reads was performed using 196 the barcode information associated with each read. The number of sorted raw sequence 197 reads ranged from 1.6 million reads (Hindenburg, ApeKI) to 3.0 million reads (Russet 198 Burbank, Pstl/Mspl). On average, 72.1 and $75.4 \%$ of the reads were successfully 199 mapped to the potato reference genome v.4.03.

201 In the diploid genotyping mode, using the IGST-GBS pipeline, a total of 30,605 202 polymorphisms (SNPs and indels) were identified from Set A using the ApeKI library 203 after removing markers having more than $12.5 \%$ missing data and a minor allele 204 frequency below $10 \%$. Indels represented $3.2 \%$ of the total, while markers with more 205 than two alleles accounted for a very small proportion $(0.3 \%)$ of polymorphisms (Table 206 2). Similarly, the Pstl/Mspl library yielded a total of 13,584 polymorphisms with indels 207 representing $4.1 \%$ of the total and markers with more than two alleles accounting for $2080.8 \%$ of polymorphisms. In the tetraploid genotyping mode, using the relaxed filter, a 
209 total of 22,946 polymorphisms (SNPs, indels, multi-nucleotide polymorphisms and

210 complex events) were identified from Set A within the ApeKI library after removing 211 markers with more than $12.5 \%$ missing data or a minor allele count below 2 . Indels

212 represented $3.9 \%$ of the total, while multi-nucleotide polymorphisms and complex events 213 represented $3.8 \%$ of the total. Markers with more than two alleles accounted for $3.6 \%$ of 214 polymorphisms. The Pstl/Mspl library yielded a total of 12,047 polymorphisms with 215 indels representing $4.8 \%$ of the total while multi-nucleotide polymorphisms and complex 216 events represented $4.1 \%$ of the total. Three- and 4 -allele markers accounted for $3.8 \%$ of 217 polymorphisms.

219 The impact of the tolerance towards missing data on the total number of markers and 220 mean read depth was then examined. For Set A, in diploid mode, the smallest number of 221 markers $(15,615)$ was obtained using the Pstl/Mspl enzyme combination without missing 222 data (Fig. 1a). Under these conditions mean read depth per scored genotype was 112.5 223 (Fig. 2a). When tolerating up to 50\% missing data, the number of SNP markers rose by $22459 \%$ (to 24,817 markers), while mean read depth per scored genotype decreased only 225 by $18 \%$ (91.7). Compared to $P$ stl $/ \mathrm{Mspl}$, when keeping only markers without missing 226 data, ApeKI yielded 27,263 markers with a mean read depth of 33.3 (Fig. 1a and 2a). 227 When tolerating up to $50 \%$ missing data, the ApeKI restriction enzyme yielded three 228 times more markers $(74,308)$ than $P$ stl/Mspl with a read depth per scored genotype 3.5 229 times lower (26.0).

231 A similar trend was observed in tetraploid mode with both relaxed and stringent filtration. 232 Under the basic filter condition (minimum of 11 reads required to call genotypes), the 
233 greatest number of markers $(40,199)$ was obtained using the restriction enzyme ApeKI 234 while tolerating up to $50 \%$ missing data per marker (Fig. 1b). A mean depth of 24.9 per 235 scored genotype was recorded under these conditions (Fig. 2b). When keeping only 236 markers with no missing data, the total number of markers decreased to 15,336 , while 237 mean marker depth increased moderately to 30.6. The Pstl/Mspl enzyme combination

238 yielded 2.7 times fewer markers than ApeKI $(14,815)$ with four times higher depth 239 coverage (100.2) when tolerating up to $50 \%$ missing data. When keeping only markers 240 with no missing data, the number of markers only decreased slightly $(10,324)$ while 241 mean marker depth increased moderately (118.7).

243 The impact of enzyme choice and tolerance to missing data was more striking under the 244 stringent filter condition where 53 reads were needed to discriminate the three 245 heterozygous genotype classes or 11 required to call a homozygote). Here, the greatest 246 number of markers $(9,148)$ was obtained using the restriction enzyme $\mathrm{Pstl} / \mathrm{Mspl}$ while 247 tolerating up to $50 \%$ missing data per marker (Fig. 1C). A mean depth of 144.1 reads per 248 scored genotype was recorded under these conditions. When keeping only markers with 249 no missing data, the total number of markers dropped by one third $(6,024$ markers), 250 while mean marker depth increased slightly (169.5) (Fig. 2c). The ApeKI enzyme yielded 251 only 1,621 markers with a depth of coverage of 62.0 when tolerating up to $50 \%$ missing 252 data (Fig. 1c). When keeping only markers with no missing data, the number of markers 253 was reduced to only 199 while mean marker depth increased to 98.5 . 


\section{Genotyping a collection of $\mathbf{3 7 5}$ clones}

256 A second series of analyses was conducted using ApeKI and the diploid calling mode on

257 a collection of 375 clones (Set B) representing the extent of diversity present in a public 258 potato breeding program in the province of Quebec (Canada). The purpose of the 259 analysis was to compare SNP calls made using GBS and the Infinium 8303 Potato Array 260 and then to conduct phylogenetic as well as association analyses. For Set B, 261 sequencing of the four ApeKI GBS libraries on an Illumina HiSeq2000 yielded 262 approximately 670.3 million reads. GBS failed to yield a sufficient number of reads 263 ( $<100 \mathrm{k}$ reads) for one clone; it was removed from the ensuing analyses. The number of 264 sorted raw sequence reads ranged from 0.37 million reads to 2.55 million reads with a 265 mean of 1.79 million reads. On average, $73.2 \%$ of the reads were successfully mapped 266 to the potato reference genome.

267 The IGST-GBS pipeline first yielded a total of $1,514,110$ markers in diploid mode. To 268 obtain a high-quality dataset, genotype calls supported by less than 11 reads, indels and 269 markers with more than $20 \%$ missing data were excluded. After applying these filters, a 270 total of 42,786 SNPs remained. As this collection of clones included ten wild relatives of 271 potato, we then examined the impact of filtration against rare alleles on the dataset. 272 Eliminating markers with a minor allele frequency below $1 \%$ or $5 \%$ yielded 22,545 and 27315,424 SNPs, respectively. The distribution of these SNPs was similar for all 274 chromosomes and is illustrated in supplemental figure 1. It proved to be highest in gene275 rich terminal regions and lowest in highly repetitive centromeric and pericentromeric 276 regions of chromosomes. The number of SNPs varied from one chromosome to another, 277 but was only moderately correlated with the physical length of the chromosome 
$278\left(r^{2}=0.15\right)$. Chromosome 10 yielded the smallest number of SNPs (767) while 279 chromosome 1 yielded the largest number of SNPs $(1,978)$ (Table 3$)$. The frequency of 280 SNPs on the twelve potato chromosomes averaged 21.1 SNPs/Mb.

282 Figure 3 shows the distribution of genotypic classes within the catalogue of SNPs called 283 among Set B. Among the called genotypes, the vast majority were homozygous for the 284 reference allele (44.8\%) or heterozygous (40.5\%), whereas $5.9 \%$ were homozygous for 285 the alternate allele and $8.8 \%$ were missing.

\section{Comparison of GBS and Infinium 8303 Potato Array SNP calls}

288 To establish the accuracy of GBS-derived genotype calls, a comparison with data 289 obtained using the SolCAP 8303 SNP Infinium array was conducted. From the initial 290 collection (374 clones), a subset of fifty-two genotypes (Set B2) was selected for this 291 purpose. Diploid genotype calls for these clones were made based on a minimum of 11 292 reads/genotype call while filtering out markers with more than $20 \%$ missing data and 293 keeping all informative markers (i.e. with at least one alternate allele). Such conditions 294 yielded a total of 45,153 markers of which 126 were in common with the Infinium $8 \mathrm{~K}$ 295 array. After removing 728 missing data in the GBS or $8 \mathrm{~K}$ array data, the actual number 296 of data points available for comparison was 5,824 . Amongst these, the match rate 297 between genotype calls was $90.4 \%$. For homozygotes, the match rate was $98.2 \%$, while 298 it dropped to $84.6 \%$ for heterozygotes. Most of the 560 discordant genotype calls 299 (83.6\%) occurred when GBS did not detect an alternate allele (Table 4). 
301 To assess if this degree of marker coverage could adequately capture the underlying

302 haplotypes and make it possible to accurately impute missing data, we performed 303 imputation of missing information on the GBS-derived set of SNPs. Imputed genotypes 304 (formerly missing data) at the 126 SNP loci common between the three GBS-derived 305 datasets and the 8K array data were then compared. As shown in Table 4, there was a 306 slight decrease in the accuracy of these imputed data compared with GBS-called SNPs, 307 with an accuracy of $84.2 \%$ over all SNPs. Thus, globally imputation of GBS data in 308 diploid mode proved challenging in this polyploid crop.

309 In addition, a comparison between data obtained through GBS or the SolCAP 8303 SNP 310 Infinium array was conducted for genotypes called in tetraploid mode. Again, a subset of 311 fifty-two genotypes from the 374-clone collection (Set B2) was selected for this purpose. 312 Tetraploid genotype calls for the 52 clones were made based on the relaxed filter 313 requiring 11 reads per clone to call all genotypic classes while filtering out markers with 314 more than $20 \%$ missing data and keeping all informative markers (i.e. with at least one 315 alternate allele). Such conditions yielded a total of 16,884 GBS-derived markers of which 31672 were in common with the 3,763 SNPs called in tetraploid mode using the Infinium 8K 317 array. After removing 480 missing data in the GBS or $8 \mathrm{~K}$ array data and 21 data where a 318 third allele was detected with GBS, the actual number of data points available for 319 comparison was 3,243 . Amongst these, the match rate between genotype calls was $32081.3 \%$. Most of the 606 discordant genotype calls $(91.4 \%)$, occurred when the two 321 genotyping tools differed slightly in their assessment of allele dosage, for example 322 monoplex vs duplex or duplex vs triplex (data not shown). 


\section{Genome-wide association mapping in potato}

325 The genetic structure of the 374 clones of Set B was explored using diploid data after

326 removing genotype calls supported by fewer than 11 reads, markers with $>20 \%$ missing

327 data and with a MAF $<5 \%$. In total 15,424 SNPs were available to perform this analysis.

328 fastStructure analysis determined the optimal value of $\mathrm{K}$ to be 5 and was used to

329 generate bar plots to visualize proportions of admixture (Fig. 4). Clones with pigmented

330 skin were clustered in subgroup 1, all of the wild species and genetic stocks were found

331 in subgroup 2, whereas most clones known to belong to a specific market class (yellow,

332 round table white and French fry processing, chip processing) were found in subgroups

3333,4 and 5 , respectively.

334

335 Then, to demonstrate the utility of dense SNP coverage as achieved here using GBS, 336 we performed GWAM on a subset of Set B composed of 318 potato lines for which

337 flower color had been determined. A total of 17,392 SNPs were selected for GWAM after 338 removing genotype calls supported by less than 11 reads and excluding SNPs with $339>20 \%$ missing data and with a MAF $<5 \%$. To reduce false positive signals, population

340 structure (covariate Q) and estimates of relatedness among individuals (covariate K) 341 were incorporated as covariates in the MLM. In this case, the most likely value for $\mathrm{K}$ was 342 found to be 4 , as wild accessions had been removed when creating this subset. This 343 model was tested on the flower colour trait and a quantile-quantile plot was produced to 344 assess the extent to which the analysis produced more significant results than expected 345 by chance (supplemental figure 2). A single highly significant region of marker-trait 346 association was seen on chromosome 2, with the peak SNP showing a $p$-value as low 
347 as $2.00 \times 10^{-8}$ (Fig. 5, Supplemental Table 1). In total, 5 significantly associated SNPs 348 spanned a region of approximately $69 \mathrm{~kb}$ (between 40.29 and $40.35 \mathrm{Mb}$ on St02), 349 encompassing eight genes, among which the dihydroflavonol 4-reductase locus 350 (40293862 to 40297510) (Supplemental Table 2). Two additional, but much weaker, 351 associations were detected on chromosomes 5 and 8.

352

353 Discussion

354

355 Choice of GBS protocol

356 Genotyping-by-sequencing is a versatile complexity reduction approach because it 357 makes it possible to focus a given sequencing effort on a fraction of the genome in such 358 a way that a higher read depth can be achieved in these regions. The required depth of 359 coverage will depend on the level of genotyping precision required. In the case of 360 tetraploid potato, a simplified genotypic classification wherein only three classes are 361 called ("diploid mode"; $A A, A B, B B$ ) can be achieved with as few as 11 reads at each 362 SNP locus. This number represents the number of reads of a single type (allele) that is 363 sufficient to confidently call a homozygote (Simko 2004). In contrast, a full 364 characterization into all five possible genotypic classes ("tetraploid mode"; AAAA, AAAB, $365 \mathrm{~A} A \mathrm{BB}, \mathrm{ABBB}$ and $\mathrm{BBBB}$ ) requires a much greater depth of coverage. In this work, we 366 estimated that 53 reads were sufficient to distinguish between the five expected 367 genotypic classes based on a Chi-square distribution. This is similar to the conclusions 368 drawn by Uitdewilligen et al. (2013), who concluded that read depths of $\sim 60-80 \mathrm{X}$ could 369 be used as a lower boundary for reliable assessment of allele copy number of sequence 
370 variants in autotetraploids. It stands to reason that different degrees of complexity

371 reduction may be needed to achieve these different levels of read depth.

372 Here, two restriction enzymes were compared, a five-base cutter (ApeKI) and an

373 enzyme pair comprising a six-base (Pstl) and a four-base cutter (Mspl). It was expected

374 that the five-base cutter would yield more markers than the six-base cutter at the

375 expense of a lower read depth per scored marker. In diploid calling mode, ApeKI yielded

376 three times more markers than Pst//Mspl $(74,308$ vs 24,817$)$ when tolerating up to $50 \%$

377 missing data. Under these conditions, the mean read depth per scored marker was 3.5

378 times lower using ApeKI instead of Pstl/Mspl (26.0 vs 91.7 ), but was still high relative to

379 the minimum number of reads required to confidently distinguish between homozygotes

380 and all types of heterozygotes (11 reads). Under the most demanding conditions, aiming

381 to determine allele dosage, the $\mathrm{Pstl} / \mathrm{Mspl}$ protocol yielded 5.6 times more markers than

382 ApeKI $(9,148$ vs 1,621$)$ when tolerating up to $50 \%$ missing data. On average, 2.3 times

383 more reads per scored genotype (144.1 vs 62.0$)$ were obtained under these conditions.

384 Thus, under conditions used in this study, Pstl/Mspl should be preferred over ApeKI to 385 call genotypes in tetraploid mode, while the ApeKI protocol maximizes the number of 386 SNP loci that can be called in diploid mode.

\section{GBS genotype calls are accurate}

389 Assessing the quality of GBS genotype calls is of paramount importance for the adoption 390 of such an approach in a new species. In this work, to assess the accuracy of our 391 genotype calls, we compared genotypes called for the same potato clones at loci in 392 common with the Infinium 8303 Potato Array SNP (Hirsch et al. 2013). This comprised a 393 set of 126 SNPs and 52 genotypes that were common to both studies. In diploid mode, 
394 after removing 728 missing data in the GBS or $8 \mathrm{~K}$ array data, the actual number of data

395 points available for comparison was 5,824 . In the absence of imputation of missing data, 396 the match rate between genotype calls was $90.4 \%$. As data imputation has been 397 demonstrated to provide highly accurate genotype calls in diploid soybean, 398 (Torkamaneh and Belzile 2015) we wanted to explore if it could be used successfully in 399 potato as well. This turned out not to be the case, as the proportion of concordant 400 genotype calls fell to $84.1 \%$. This is likely due to an insufficient marker coverage leading 401 to incomplete capture of haplotype diversity. Although a relatively large number of 402 markers was available to perform missing data imputation (45k), haplotype diversity in a 403 tetraploid species such as potato tends to be quite high (Uitdewilligen et al. 2013). 404 Accuracy of missing data imputation has been shown to decline markedly when marker 405 coverage is insufficient (Torkamaneh and Belzile 2015). This result is lower than those 406 reported by Uitdewilligen et al. (2013): $97.9 \%$ of the homozygous and $99.9 \%$ of the 407 heterozygous GBS calls having been found concordant with the results of KASP 408 genotyping. The authors argued that their method of bait-hybridisation enrichment was 409 of great importance for genotyping accuracy because it avoided complex multicopy and 410 repetitive elements of the genome, thus circumventing potential difficulties in

411 distinguishing allelic variants form paralogous sequences. This could indeed explain the 412 lower match rate reported here. In polyploid species, SNP detection is confounded by a 413 highly similar homeologous sequence where a polymorphism between subgenomes 414 must be differentiated from a SNP (Clevenger and Ozias-Akins 2015). Almost half of the 415 S. tuberosum genome $(48.8 \%)$ is composed of repetitive elements and such complex 416 elements are associated with $>95 \%$ of genes (Mehra et al. 2015). Moreover, extensive 417 copy number variation has been documented in potato, impacting $219.8 \mathrm{Mb}(30.2 \%)$ of 
418 its genome with nearly $30 \%$ of genes subject to at least partial duplication or deletion

419 (Hardigan et al. 2016). Nonetheless, these results are difficult to compare directly with

420 ours as this work focused on a limited set of genomic regions (803) and cannot provide

421 whole-genome coverage comparable to the one achieved above.

422 Aside from the complex nature of the potato genome, other causes may account for the

$4239.6 \%$ discordant genotype calls between GBS and 8K array in diploid mode and $18.7 \%$

424 discordant genotype calls in tetraploid mode. Some of these $(83.6 \%$, in diploid mode,

$42519.6 \%$ in tetraploid mode) occurred when GBS did not detect an alternate allele, a

426 situation referred to as allele dropout. Such events may occur if the SNP underlying the

427 alternate allele created or abolished an ApeKI, Mspl or Pstl recognition site and could

428 not be captured during the library preparation process. Other polymorphisms on raw

429 reads corresponding to a missing allele could lead to similar results. In addition, a GBS

430 haplotype corresponding to an alternate allele could map to several locations on the

431 genome and be eliminated in the GBS pipeline.

432 Lastly, the Infinium 8303 SNP array data generates variable cluster positioning and 433 quality for each SNP. Therefore, a custom cluster file was generated for each SNP 434 (Hirsch et al. 2013). In contrast GBS IGST pipeline treats all markers uniformly after

435 removing all low-quality reads during the read cleaning step (Sonah et al. 2013). This 436 sole fact could account for a sizable proportion of discrepancies between the two 437 approaches. Nevertheless, the high match rate of genotype calls support is a proof of 438 the high quality of GBS in potato.

440 Genome-wide association mapping is possible in potato 
441 The success of GWAM depends on achieving an adequate marker coverage within a set

442 of germplasm. The most recent and comprehensive study on this subject, conducted 443 using the 20K SolSTW array which comprises 14,530 SNPs, concluded that such

444 coverage should be adequate to detect sufficient QTLs (Vos et al. 2017). However it is 445 important to underline that only a fraction of the SNP loci tested by an array are 446 polymorphic within a population and show a minor allele frequency high enough to 447 perform GWAM. For instance, in the same work, the number of available SNPs within 448 genotypes released after $2004(n=255)$ was only 5,282 and 7,926 following filtering at $449 \mathrm{MAF}>10 \%$ and $\mathrm{MAF}>1 \%$, respectively.

450 In this work, the number of markers used to perform GWAM $(17,392$ SNPs) was higher 451 than what the SolSTS array could provide in a perfect situation where all SNPs would be 452 polymorphic. The analysis of the population structure allowed to group together clones 453 sharing similarities in terms of domestication (wild species and genetic stock vs 454 cultivated clones) or in terms of market classes (yellow, round table white and French fry 455 processing, chip processing), consistent with a previous study conducted using the 456 Infinium 8303 Potato Array SNP (Hirsch et al. 2013).

457 Using this dataset, we found a single strong association signal on chromosome 2 and 458 two other significant signals on chromosomes 5 and 8. Interestingly, our most significant 459 SNP on chromosome 2 at position 40,294,266 $\left(p=2.00 \times 10^{-8}\right)$ was in the middle of the 460 dihydroflavonol 4-reductase (DFR) gene and the second one at position 40,301,913 ( $p=$ $4611.07 \times 10^{-7}$ ) was located only $4.4 \mathrm{~kb}$ away. The DFR gene underlies the R locus, a key 462 enzyme in anthocyanin synthesis (Zhang et al. 2009). This concurs with a QTL reported 463 to explain over $40 \%$ of the variation for flower color and believed to correspond to the $R$ 464 locus (McCord et al. 2011). 
465 Intriguingly, a recent study where a GWAM analysis for flower colour was conducted 466 among a collection of 809 accessions did not identify markers associated to the DFR 467 gene on chromosome 2 (Berdugo-Cely et al. 2017). The $p$ values reported were slightly 468 high, as only three markers associated with flower primary color were below 0.001 . This 469 could have been caused by an insufficient marker coverage to account for linkage 470 disequilibrium on chromosome 2, since the Infinium 8303 potato SNP array yielded only 4715,968 useful markers, almost three time less than here. In addition, the mode of scoring 472 for flower colour could account for the differences between both studies, as their coding 473 for primary flower colour was on a scale from 1 to 8 while a binary code (white or any 474 colour) was used here. Globally, our ability to identify SNP markers associated to flower 475 color through GWAM is another demonstration of the usefulness of GBS in potato.

476 In this work, we showed that GBS can be successfully used for various genetic analyses

477 in autotetraploid potato. Depending on the aim of the study, different library preparation 478 protocols are available that yield different numbers of markers and depth of coverage, 479 one being achieved at the cost of the other. The resulting catalogues of SNPs are found 480 to be of good quality and were used successfully to perform useful genetic analyses 481 such as phylogenetic trees and GWAM.

483 Acknowledgments This work was funded by the Fédération des Producteurs de 484 Pomme de Terre du Québec, by the Programme de soutien à l'innovation en 485 agroalimentaire of the ministère de l'Agriculture, des Pêcheries et de l'Alimentation du 486 Québec and Agriculture and Agri-Food Canada as well as an Engage grant financed by 487 the Natural Sciences and Engineering Research Council of Canada and ProgesT2001. 


\section{References}

490 Bali S., Sathuvalli V., Brown C., Novy R., Ewing L., Debons J., et al. 2016. Genetic 491 fingerprinting of potato varieties from the northwest potato variety development program. 492 Am. J. Potato Res. 94 (1): 54-63. doi: 10.1007/s12230-016-9547-z

493 Benjamini Y., and Yekutieli D. 2005. Quantitative trait loci analysis using the false 494 discovery rate. Genetics, 171 (2): 783-790. doi: 10.1534/genetics.104.036699.

495 Berdugo-Cely J., Valbuena R.I., Sanchez-Betancourt E., Barrero L.S. and Yockteng R. 496 2017. Genetic diversity and association mapping in the Colombian central collection of 497 Solanum tuberosum L. Andigenum group using SNPs markers. PLoS One, 12 (3): 498 e0173039. doi:10.1371/journal.pone.0173039

499 Bourke P.M., Voorrips R.E., Kranenburg T., Jansen J., Visser R.G.F., and Maliepaard C. 500 2016. Integrating haplotype-specific linkage maps in tetraploid species using SNP 501 markers. Theor. Appl. Genet. 129 (11): 2211-2226. doi:10.1007/s00122-016-2768-1

502 Bradbury P.J., Zhang Z., Kroon D.E., Casstevens T.M., Ramdoss Y., and Buckler, E.S. 503 2007. TASSEL: Software for association mapping of complex traits in diverse samples.

504 Bioinformatics, 23 (19): 2633-2635. doi: 10.1093/bioinformatics/btm308

505 Browning S.R., and Browning B.L. 2007. Rapid and accurate haplotype phasing and 506 missing data inference for whole genome association studies by use of localized 507 haplotype clustering. Am. J. Hum. Genet 81. (5): 1084-1097. doi:10.1086/521987

508 Clevenger J., and Ozias-Akins P. 2015. SWEEP: A tool for filtering high-quality SNPs in 509 polyploid crops. Genes Genom. Genet. 5 (9): 1797-1803. doi: 10.1534/g3.115.019703 
510 Danecek P., Auton A., Abecasis G., Albers C.A., Banks E., De Pristo M.A., et al. 2011.

511 The variant call format and VCFtools. Bioinformatics, 27 (15): 2156-2158. doi:

512 10.1093/bioinformatics/btr330.

513 Elshire R.J., Glaubitz J.C., Sun Q., Poland J.A., Kawamoto K. Buckler E.S., et al. 2011.

514 A robust, simple genotyping-by-sequencing (GBS) approach for high diversity species.

515 PLoS One, 6 (5): e19379. doi: doi:10.1371/journal.pone.0019379

516 Endelman J.B., and Jansky S. 2016. Genetic mapping with an inbred line-derived F2

517 population in potato. Theor. Appl. Genet. 129 (5): 935-943. doi: 10.1007/s00122-016$518 \quad 2673-7$

519 Endelman J.B., Schmitz Carley C.A., Douches D.S., Coombs J.J., Bizimungu B., De 520 Jong W.S., et al. 2017. Pedigree reconstruction with genome-wide markers in potato. 521 Am. J. Potato Res. 94 (2): 184-190. doi:10.1007/s12230-016-9556-y

522 Esnault F., Pellé R., Dantec J.P., Bérard A., Le Paslier M.C., and Chauvin J.É. 2016.

523 Development of a potato cultivar (Solanum tuberosum L.) core collection, a valuable tool 524 to prospect genetic variation for novel traits. Potato Res. 59 (4): 329-343. 525 doi:10.1007/s11540-016-9332-x

526 Felcher K.J., Coombs J.J., Massa A.N., Hansey C.N., Hamilton J.P., Veilleux R.E., et al. 527 2012. Integration of two diploid potato linkage maps with the potato genome sequence.

528 PLoS One, 7 (4): e36347. doi: 10.1371/journal.pone.0036347

529 Garrison E., and Marth G. 2012. Haplotype-based variant detection from short-read 530 sequencing. arXiv:1207.3907 Available from https://arxiv.org/abs/1207.3907 [accessed 53112 February 2018]. 
532 Hackett C.A., Bradshaw J.E., and Bryan G.J. 2014. QTL mapping in autotetraploids

533 using SNP dosage information. Theor. Appl. Genet. 127 (9): 1885-1904. doi:

$534 \quad 10.1007 / \mathrm{s} 00122-014-2347-2$

535 Hardigan M.A., Crisovan E., Hamilton J.P., Kim J., Laimbeer P., Leisner C.P., et al. 536 2016. Genome reduction uncovers a large dispensable genome and adaptive role for

537 copy number variation in asexually propagated Solanum tuberosum. Plant Cell, 28 (2):

538 388-405. doi: $10.1105 /$ tpc. 15.00538

539 Hirsch C.N., Hirsch C.D., Felcher K., Coombs J., Zarka D., Van Deynze A., et al. 2013.

540 Retrospective View of North American Potato (Solanum tuberosum L.) Breeding in the

$54120^{\text {th }}$ and $21^{\text {st }}$ Centuries. Genes Genom. Genet. 3 (6): 1003-1013. doi:

$542 \quad 10.1534 / g 3.113 .005595$

543 Jiang N., Zhang F., Wu J., Chen Y., Hu X., Fang O., et al. 2016. A highly robust and 544 optimized sequence-based approach for genetic polymorphism discovery and 545 genotyping in large plant populations. Theor. Appl. Genet. 129 (9): 1739-1757. doi: $546 \quad 10.1007 / \mathrm{s} 00122-016-2736-9$

547 Kolech S.A., Halseth D., Perry K., Wolfe D., Douches D.S., Coombs J., et al. 2016. 548 Genetic diversity and relationship of Ethiopian potato varieties to germplasm from North 549 America, Europe and the international potato center. Am. J. Potato Res. 93 (6): 609-609.

550 doi: $10.1007 / \mathrm{s} 12230-016-9543-3$

551 Lee H., Dekkers J.C., Soller M., Malek M., Fernando R.L., and Rothschild M.F. 2002.

552 Application of the false discovery rate to quantitative trait loci interval mapping with 553 multiple traits. Genetics, 161 (2): 905-914. doi: 10.1534/genetics.104.036699 
554 Manrique-Carpintero N.C., Coombs J.J., Cui Y., Veilleux R.E., Buell C.R., and Douches

555 D. 2015. Genetic map and QTL analysis of agronomic traits in a diploid potato 556 population using single nucleotide polymorphism markers. Crop Sci. 55 (6): 2566-2579.

557 doi: $10.2135 /$ cropsci2014.10.0745

558 Massa A.N., Manrique-Carpintero N.C., Coombs J.J., Zarka D.G., Boone A.E., Kirk

559 W.W., et al. 2015. Genetic linkage mapping of economically important traits in cultivated

560 tetraploid potato (Solanum tuberosum L.). Genes Genom. Genet. 5 (11): 2357-2364. doi:

$561 \quad 10.1534 / g 3.115 .019646$

562 McCord P.H., Sosinski B.R., Haynes K.G., Clough M.E., and Yencho G.C. 2011.

563 Linkage mapping and QTL analysis of agronomic traits in tetraploid potato (Solanum

564 tuberosum subsp. tuberosum). Crop Sci. 51 (2): 771-785. doi:

$565 \quad 10.2135 /$ cropsci2010.02.0108

566 Mehra M., Gangwar I., and Shankar R. 2015. A deluge of complex repeats: The

567 Solanum genome. PLoS One, 10 (8): e0133962. doi:10.1371/journal. pone.0133962

568 Muktar M.S., Lübeck J., Strahwald J., and Gebhardt C. 2015. Selection and validation of

569 potato candidate genes for maturity corrected resistance to Phytophthora infestans

570 based on differential expression combined with SNP association and linkage mapping.

571 Front. Genet. 6: 294. doi: 10.3389/fgene.2015.00294

572 Obidiegwu J.E., Sanetomo R., Flath K., Tacke E., Hofferbert H.R., Hofmann A., et al. 573 2015. Genomic architecture of potato resistance to Synchytrium endobioticum 574 disentangled using SSR markers and the 8.3k SolCAP SNP genotyping array. BMC 575 Genet. 16: 38. doi: 10.1186/s12863-015-0195-y 
576 Poland J., Endelman J., Dawson J., Rutkoski J., Wu S., Manes Y., et al. 2012. Genomic

577 selection in wheat breeding using genotyping-by-sequencing. Plant Gen. 5 (3): 103-113.

578 doi:10.3835/plantgenome2012.06.0006

579 Raj A., Stephens M., and Pritchard J.K. 2014. fastSTRUCTURE: Variational inference of 580 population structure in large SNP data sets. Genetics, 197 (2): 573-589. doi:

$581 \quad 10.1534 /$ genetics.114.164350

582 Rak K., Bethke P.C., and Palta J.P. 2017. QTL mapping of potato chip color and tuber 583 traits within an autotetraploid family. Mol. Breed. 37 (2): 15. doi:10.1007/s11032-017$584 \quad 0619-7$

585 Sharma S.K., Bolser D., de Boer J., Sønderkær M., Amoros W., Federico Carboni M., et

586 al. 2013. Construction of reference chromosome-scale pseudomolecules for potato: 587 integrating the potato genome with genetic and physical maps. Genes. Genom. Genet. 3 588 (11): 2031-2047. doi: 10.1534/g3.113.007153

589 Simko I. 2004. One potato, two potato: Haplotype association mapping in 590 autotetraploids. Trends Plant Sci. 9 (9): 441-448. doi: 10.1016/j.tplants.2004.07.003

591 Sonah H., Bastien M., Iquira E., Tardivel A., Légaré G., Boyle B., et al. 2013. An 592 improved genotyping by sequencing (GBS) approach offering increased versatility and 593 efficiency of SNP discovery and genotyping. PLoS One, 8 (1): e54603. 594 doi:10.1371/journal.pone.0054603

595 Storey J.D., and Tibshirani R. 2003. Statistical significance for genomewide studies. 596 Proc. Natl. Acad. Sci. 100 (16): 9440-9445. doi: 10.1073/pnas.1530509100 
597 Sverrisdóttir E., Byrne S., Sundmark E.H.R., Johnsen H.Ø., Kirk H.G., Asp T., et al.

598 2017. Genomic prediction of starch content and chipping quality in tetraploid potato 599 using genotyping-by-sequencing. Theor. Appl. Genet. 130 (10): 2091-2108. doi: $60010.1007 / \mathrm{s} 00122-017-2944-y$

601 Tang Y., Liu X., Wang J., Li M., Wang Q., Tian F., et al. GAPIT version 2: an enhanced 602 integrated tool for genomic association and prediction. Plant Genome, 9 (2) doi: 603 10.3835/plantgenome2015.11.0120

604 Torkamaneh D., and Belzile F. 2015. Scanning and filling: ultra-dense SNP genotyping 605 combining genotyping-by-sequencing, SNP array and whole-genome resequencing 606 data. PLoS One, 10 (7): e0131533. doi:10.1371/journal.pone.0131533

607 Uitdewilligen J.G.A.M.L., Wolters A.M.A., D'hoop B.B., Borm T.J.A., Visser R.G.F., and 608 van Eck H.J. 2013. A next-generation sequencing method for genotyping-by-sequencing 609 of highly heterozygous autotetraploid potato. PLoS One, 8 (5): e62355. 610 doi:10.1371/journal.pone.0062355

611 Vos P.G., Paulo M.J., Voorrips R.E., Visser R.G.F., van Eck H.J., van Eeuwijk F.A. 612 2017. Evaluation of LD decay and various LD-decay estimators in simulated and SNP613 array data of tetraploid potato. Theor. Appl. Genet. 130 (1): 123-135 614 doi:10.1007/s00122-016-2798-8

615 Vos P.G., Uitdewilligen J.G.A.M.L., Voorrips R.E., Visser R.G.F., and van Eck H.J. 2015. 616 Development and analysis of a 20K SNP array for potato (Solanum tuberosum): an 617 insight into the breeding history. Theor. Appl. Genet. 128 (12): 2387-2401. doi: $618 \quad 10.1007 / s 00122-015-2593-y$ 
619 Zhang Y., Cheng S., De Jong D., Griffiths H., Halitschke R., and De Jong W. 2009. The 620 potato R locus codes for dihydroXavonol 4-reductase. Theor. Appl. Genet. 119 (5): 931 621 937. doi: 10.1007/s00122-009-1100-8

622 
623 Table 1 Summary of sequenced raw and processed reads in eight potxato genotypes 624 obtained on an Illumina HiSeq2000

625

\begin{tabular}{lcccccc} 
& \multicolumn{2}{c}{ Raw (Millions) } & \multicolumn{2}{c}{ Processed (Millions) } & \multicolumn{2}{c}{ Processed (\%) } \\
& ApeKI & Pstl/Mspl & ApeKI & Pstl/Mspl & ApeKI & Pstl/Mspl \\
\hline Argos & 2.1 & 2.9 & 1.5 & 2.2 & 71.2 & 74.0 \\
Conestoga & 2.4 & 2.5 & 1.7 & 1.8 & 71.5 & 74.1 \\
Divina & 2.9 & 2.4 & 2.1 & 1.8 & 71.6 & 76.0 \\
Envol & 2.7 & 2.3 & 2.0 & 1.7 & 72.0 & 74.7 \\
Eva & 2.4 & 2.2 & 1.7 & 1.7 & 72.0 & 75.1 \\
Hindenburg & 1.6 & 1.9 & 1.2 & 1.4 & 72.7 & 75.2 \\
Roselys & 1.9 & 2.4 & 1.3 & 1.8 & 71.7 & 75.8 \\
Russet Burbank & 3.0 & 3.0 & 2.2 & 2.4 & 73.9 & 78.5 \\
\hline Average & 2.5 & 2.5 & 1.7 & 1.9 & 72.1 & 75.4
\end{tabular}


627 Table 2 Summary of markers among eight potato genotypes with two restriction enzyme 628 combinations and two calling modes for markers sustained by less than $12.5 \%$ missing 629 data and having a minor allele frequency lower than $10 \%$ (diploid) or a minor allele count 630 (tetraploid) lower than three

631

\begin{tabular}{rrrr}
\hline \multicolumn{2}{c}{ IGST } & \multicolumn{2}{c}{ Freebayes } \\
Diploid mode & \multicolumn{2}{c}{ Tetraploid mode } \\
ApeKI & PstI/Mspl & ApeKI & PstI/Mspl \\
\hline 30,605 & 13,584 & 22,946 & 12,047 \\
29,625 & 13,027 & 21,181 & 10,970 \\
$96.8 \%$ & $95.9 \%$ & $92.3 \%$ & $91.1 \%$ \\
980 & 557 & 904 & 584 \\
$3.2 \%$ & $4.1 \%$ & $3.9 \%$ & $4.8 \%$ \\
$/$ & $/$ & 861 & 493 \\
$/$ & $/$ & $3.8 \%$ & $4.1 \%$ \\
\hline
\end{tabular}

632 
633 Table 3 Number of SNPs detected per chromosome by GBS among a collection of 374 634 clones in diploid mode after removing genotype calls supported by less than 11 reads, 635 indels, markers with more than $20 \%$ missing data or with a minor allele frequency inferior 636 to $5 \%$

637

\begin{tabular}{ccc}
\hline CHR & Length (Mb) & Nb of SNPs \\
\hline 1 & 82.6 & 1978 \\
2 & 45.3 & 1539 \\
3 & 57.2 & 1697 \\
4 & 66.3 & 1361 \\
5 & 49.5 & 1169 \\
6 & 55.1 & 1133 \\
7 & 52.9 & 1244 \\
8 & 52.4 & 1079 \\
9 & 57.3 & 1288 \\
10 & 56.0 & 767 \\
11 & 42.5 & 1061 \\
12 & 57.4 & 1108 \\
\hline Total & 674.4 & 15,424
\end{tabular}

638

639

640 
641 Table 4 Comparison of GBS and SolCAP 8K array SNP calls in diploid mode with and 642 without imputation

643

\begin{tabular}{crrr}
\hline \multicolumn{2}{c}{ Genotype call } & \multicolumn{2}{c}{ Data } \\
solCAP & GBS & Original & Imputed \\
\hline $0 / 0$ & $0 / 0$ & 2237 & 2430 \\
$0 / 1$ & $0 / 1$ & 2823 & 2902 \\
$1 / 1$ & $1 / 1$ & 204 & 31 \\
$1 / 1$ & $0 / 0$ & 45 & 240 \\
$0 / 1$ & $0 / 0$ & 417 & 644 \\
$1 / 1$ & $0 / 1$ & 6 & 8 \\
$0 / 0$ & $0 / 1$ & 50 & 57 \\
$0 / 1$ & $1 / 1$ & 41 & 38 \\
$0 / 0$ & $1 / 1$ & 1 & 20 \\
\hline Total concordant calls & 5264 & 5363 \\
Total disconcordant calls & 560 & 1007 \\
Total nb. of datapoints & 5824 & 6370
\end{tabular}

644

645

646

647

648

649

650 
651 Fig. 1 Number of markers according to percentage missing data in a) diploid mode, b)

652 tetraploid mode under relaxed filter conditions and c) tetraploid mode under stringent 653 filter conditions. Relaxed filter requires 11 reads to call all genotypic classes while 654 stringent filter requires 11 reads to call homozygotes (AAAA \& BBBB) and 53 reads to 655 distinguish the heterozygotes (AAAB, AABB \& ABBB) 656

657 Fig. 2 Depth of coverage per scored genotype in a) diploid mode, b) tetraploid mode 658 under relaxed filter conditions and c) tetraploid mode under stringent filter conditions 659 according to percentage missing data. Relaxed filter requires 11 reads to call all 660 genotypic classes while stringent filter requires 11 reads to call homozygotes (AAAA \& 661 BBBB) and 53 reads to distinguish the heterozygotes (AAAB, AABB \& ABBB)

663 Fig. 3 Distribution of genotypes calls within the 374 clones collection amongst 15,424 664 SNPs with less than $20 \%$ missing data and a minor allele frequency greater than $5 \%$. A 665 represents the reference allele and B represents the alternate allele

667 Fig. 4 Graphical display of population substructure for 374 lines based on 15,424 SNP 668 markers with diploid genotype calls and $K=5$ subpopulations

670 Fig. 5 Genome-wide association scan for flower pigmentation. The $-\log _{10}(p)$ values 671 from the genome-wide scan are plotted against the single nucleotide polymorphism 672 (SNP) positions on the physical map of each chromosome. The horizontal line shows 673 the threshold corresponding to a false discovery rate $<0.10$ 


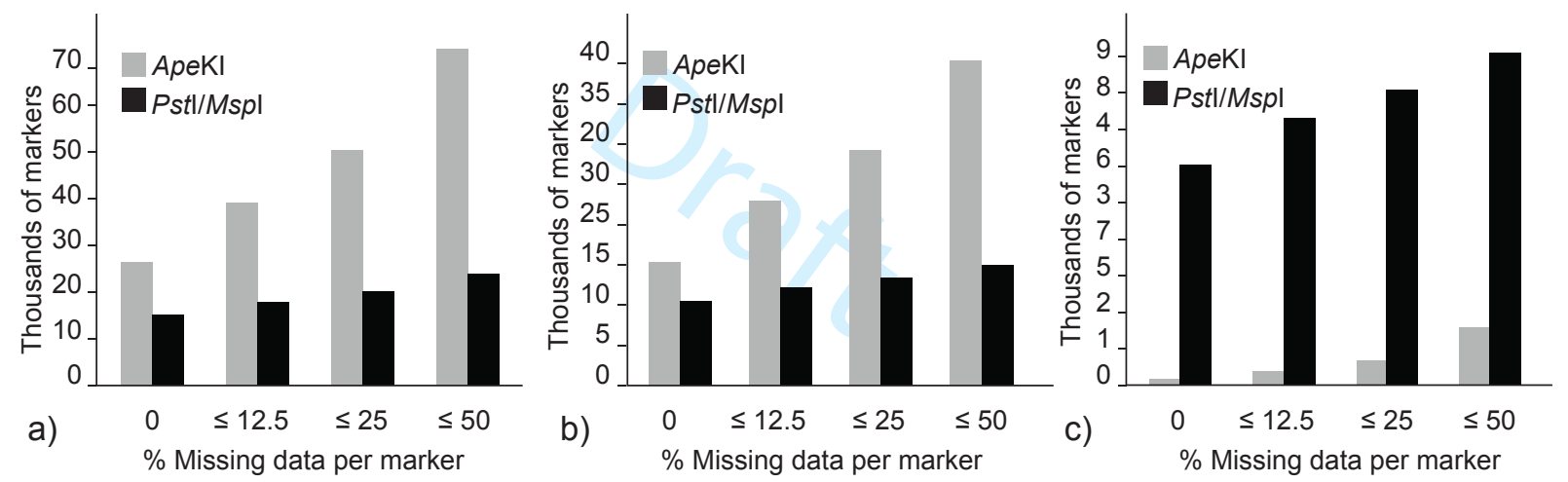



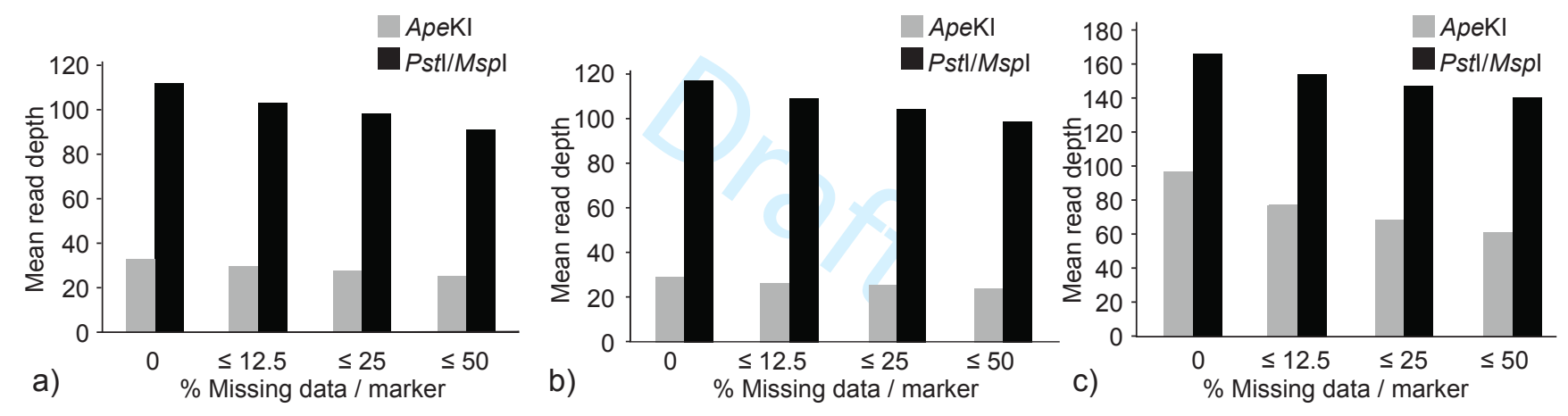


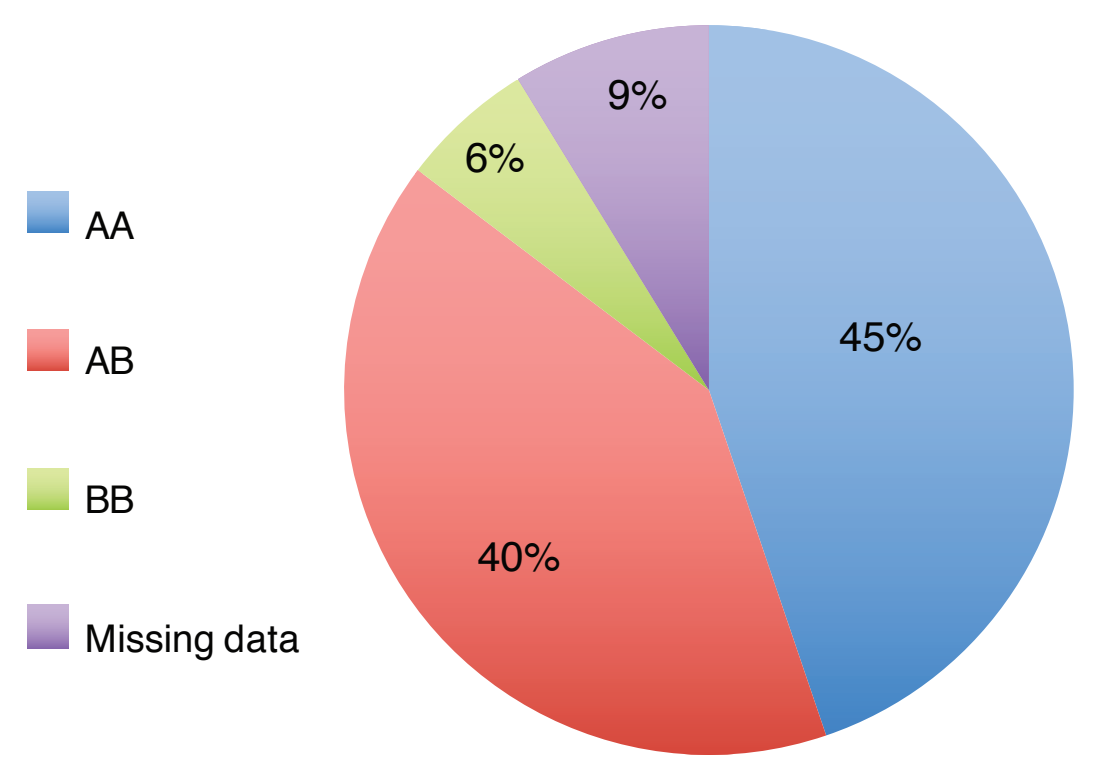




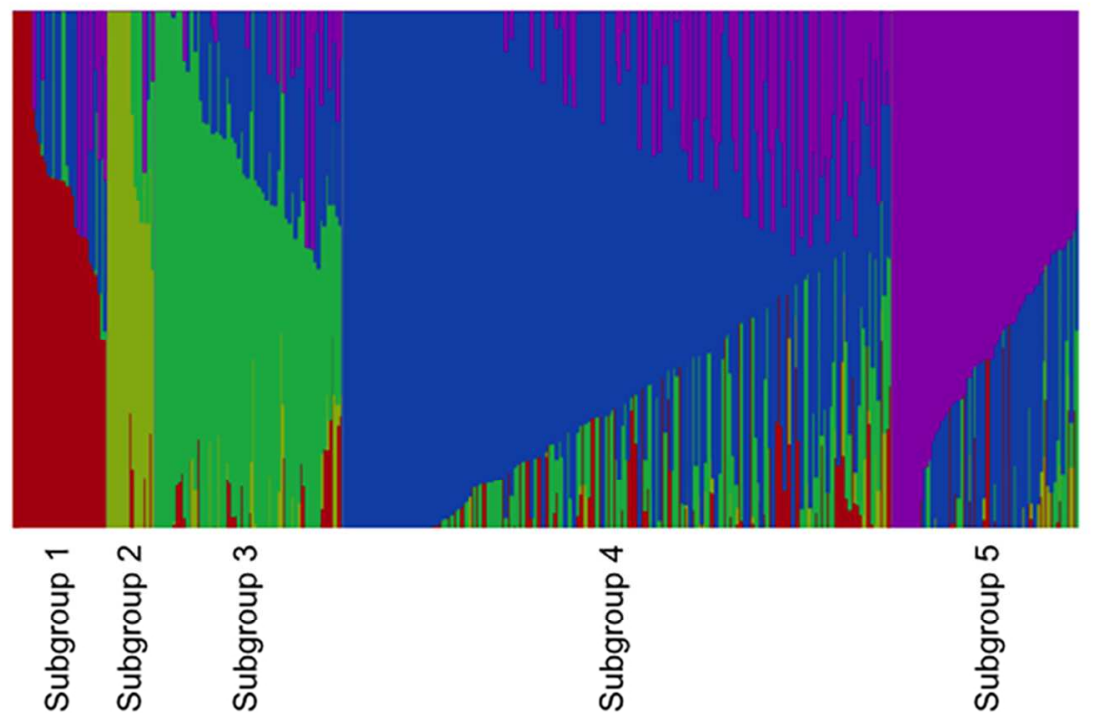

$101 \times 76 \mathrm{~mm}(300 \times 300$ DPI $)$ 


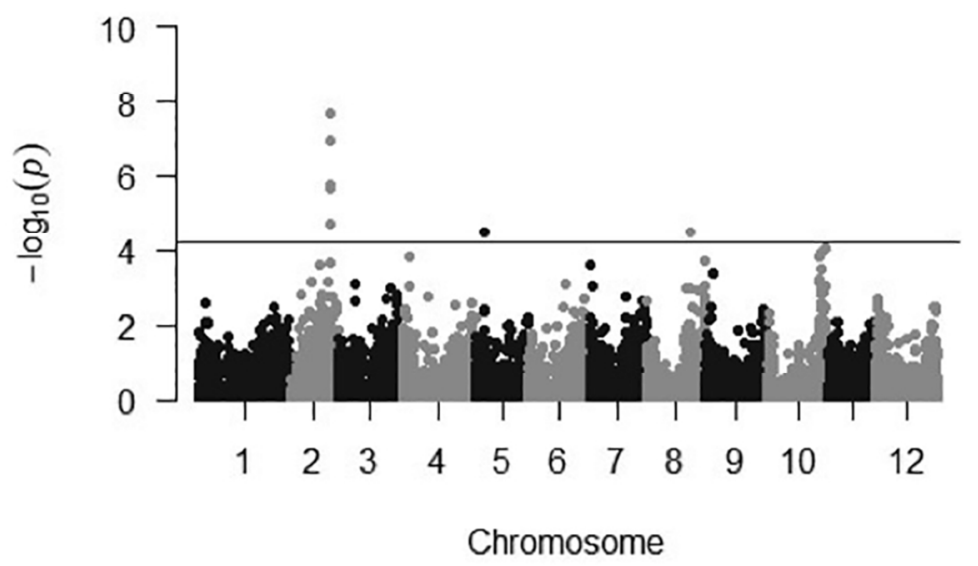

$101 \times 76 \mathrm{~mm}(300 \times 300 \mathrm{DPI})$ 